\title{
Typing Local Control and State Using Flow Analysis
}

\author{
Arjun Guha, Claudiu Saftoiu, and Shriram Krishnamurthi \\ Brown University
}

\begin{abstract}
Programs written in scripting languages employ idioms that confound conventional type systems. In this paper, we highlight one important set of related idioms: the use of local control and state to reason informally about types. To address these idioms, we formalize run-time tags and their relationship to types, and use these to present a novel strategy to integrate typing with flow analysis in a modular way. We demonstrate that in our separation of typing and flow analysis, each component remains conventional, their composition is simple, but the result can handle these idioms better than either one alone.
\end{abstract}

\section{Introduction}

"Scripting" languages are widely used in software development. Their lack of static types is touted as a positive feature that enables rapid prototyping. As programs grow large and complex, programmers need tools to reason about their code. A retrofitted type system, which would provide additional static guarantees, would help programmers manage evolution. However, care must be taken so that common idioms aren't deemed untypable; otherwise, either many programs or the languages themselves would have to change.

In section 2, we present examples from the aforementioned scripting languages that make heavy use of control and state to reason about "types". In section 3 , we introduce a core calculus that can express the essence of these examples and a simple type system for this core calculus; but this type system alone cannot type-check our examples. In section [5, we present a program analysis that can reason about the idioms in our examples. One of our contributions is to clarify the relationship between static types and runtime tags (section 4), which scripting languages often confuse; we exploit this relationship to integrate type-checking with flow analysis in a tractable manner. In section [6, we present a simple proof of soundness for the combination of typing and flow analysis.

We have built an experimental type checker for JavaScript that uses these ideas. The implementation, discussion, and elided proofs, are available from http://www.cs.brown.edu/research/plt/dl/flowtyping/v1/.

\section{Control and State in Scripting Languages}

We consider examples from canonical scripting languages. Our first example is the JavaScript function in figure 1, which serializes arbitrary values to strings. 


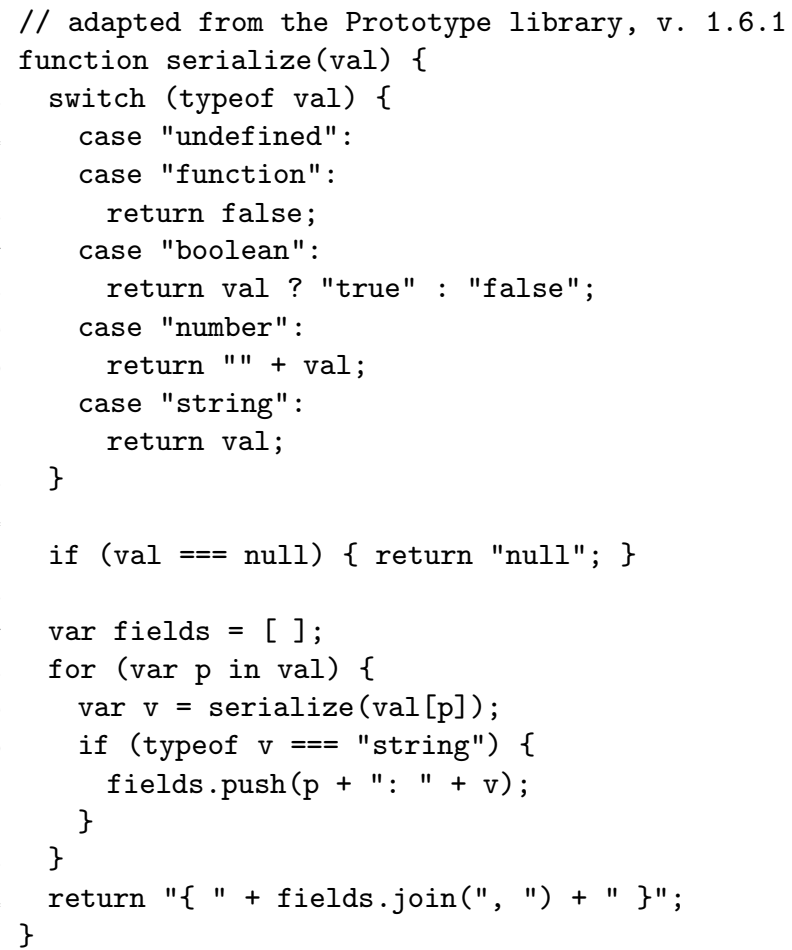

Fig. 1. Non-local control in JavaScript

Functions and the special value undefined cannot be serialized, so for these it returns false. Since val may be any value, in a typed dialect of JavaScript, serialize should have a type equivalent to $T \rightarrow \operatorname{Str} \cup$ Bool.

Let us informally reason about the type-safety of serialize. On line 3, the function branches on the result of typeof val. In JavaScript, the typeof operaton returns a string representing the "runtime type" of its argument. Thereafter:

- For case "undefined", control falls through to line 5.

- On line 5, for case "function", the function returns false.

- On line 7, for case "boolean", the function branches on val and returns either "true" or "false". val is a boolean because none of the preceding cases fall through to line 7.

- On line 9, for case "number", the function uses string concatenation to coerce the number val to a string. Thus, val is a number because none of the preceding cases fall through to line 9 .

- On line 11, for case "string", the function returns val. Thus, val is a string because none of the preceding cases fall through to here.

${ }^{1}$ To be precise, typeof does not return a (static) type but a (runtime) tag. This distinction becomes significant in section 4 


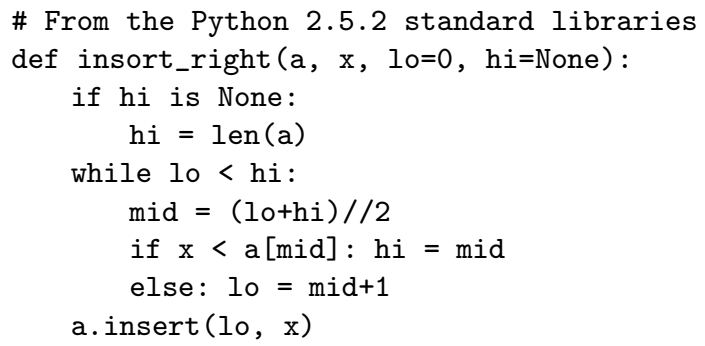

Fig. 2. Heap-sensitive reasoning in Python

This switch is missing a case! If typeof val === "object", none of the earlier cases match and control will fall through. However, since all the explicitly handled cases return, we know that typeof val === "object" holds on lines 15-24.

JavaScript has a value null and typeof null === "object". Therefore, line 15 tests for null and if the test is true, the program returns "null". However, if the test is false, because the conditional does not have a false-branch, control proceeds to line 17 . Since the true-branch returns, val !== null holds on lines 17-24. We can safely use val as an object on these lines. Lines 20 and 21 also employ flow-directed reasoning, but are relatively trivial. Therefore, we can conclude that serialize is safe.

Heap-Sensitive Reasoning. Let us consider a Python example. The function insort_right (figure 2) inserts the argument $\mathrm{x}$ into the sorted array a, preserving sortedness. The additional optional arguments, lo and hi, are expected to be integers that specify the portion of array a to be returned.

The intended defaults are $10=0$ and $h i=l e n(a)$. However, the values of other arguments are not in scope when these expressions are evaluated. Therefore, hi=len(a) would signal an unbound identifier error. Instead, the program uses the default hi=None (which better guards against premature use than would a numeric default like 0 ). The test on line 3 and the side-effect on line 4 ensure that $\mathrm{hi}$ is an integer in the continuation of the if-statement (lines 5-9). This function relies not only on control-flow, but on the interaction of control and state to reason about types.

Dynamic Dispatch and Type Tests. We reasoned about the use of serialize and insort_right by following their convoluted control-flow and side-effects, instead of merely following their syntactic structure. A reader may argue that these functions are "bad style", so a type system can legitimately reject them. For example, an easily typable alternative to serialize is to extend the builtin prototypes (Object, String, etc.) with a serialize method and rely on dynamic dispatch, instead of reflection. Unfortunately, extending builtin classes runs into the fragile base class problem [17] and is thus considered bad practice (e.g., 6]).

Irrespective of these options, the code above reflects what programmers do in practice. Figure 3 offers a conservative estimate of the prevalence of type 


\begin{tabular}{|l|r|r|r|r|r|}
\hline Checks For & JS Gadgets & Python stdlib & Ruby stdlib & Django & Rails \\
\hline${\text { undefined } / \text { null }^{a}}^{a}$ & 3,298 & 1,686 & 538 & 868 & 712 \\
instanceof $^{b}$ & 17 & 613 & 1,730 & 647 & 764 \\
typeof $^{c}$ & 474 & 381 & & 4 & \\
field-presence $^{d}$ & & 504 & 171 & 348 & 719 \\
\hline Total Checks & 3,789 & 3,184 & 2,439 & 1,867 & 2,195 \\
\hline LOC & 617,766 & 313,938 & 190,002 & 91,999 & 294,807 \\
\hline
\end{tabular}

\footnotetext{
${ }^{a}$ None in Python, and nil in Ruby.

${ }^{b}$ isinstance in Python, and .is_a? and .instance_of? in Ruby.

${ }^{c}$ type in Python.

${ }^{d}$ hasattr in Python, and .respond_to? in Ruby.
}

Fig. 3. Tag Checks and Related Checks

tests and related checks across a broad corpus of code, by counting occurrences of type testing operators. We believe these numbers undercount, since they do not account for heap-sensitive reasoning and other type testing patterns. For example, we do not try to estimate how often JavaScript programs test for the presence of a field, because this operation is syntactically indistinguishable from field lookup.

Perspective. The examples above make heavy use of local control and state to reason informally about "types". A static type system that admits these programs will need to support this style of reasoning and various other features (e.g., objects). The book-keeping needed to account for control and state can pervade the entire type system and occlude its typing of other features. Our novel flow typing system therefore separates typing from the account of flows and state. We present flow typing for an explicitly typed core calculus. We view type inference as a programmer convenience [9] that we leave for future work.

\section{Semantics and Types}

To present formal type and flow analysis systems, we have to settle on a runtime semantics. The languages under consideration have a kernel of higher-order functions and state that is essentially the same. This kernel is almost sufficient for our presentation, but we need to pick control operators and primitive operators, which do vary between languages. For now we will pick operators based on JavaScript, and return to this issue in section 5 .

Figure 4 specifies the syntax and semantics of $\lambda_{S}$, which is a core calculus that is sufficient for our exposition of flow typing. $\lambda_{S}$ includes higher-order functions, mutable references, conditionals, a control operator (break), and JavaScriptinspired primitives. Type annotations (discussed below) are ignored during evaluation. Although $\lambda_{S}$ has first-class references, note that JavaScript, Python, and Ruby do not. However, first-class references allow us to model mutable variables and stateful objects [8, Section 2.1]. 


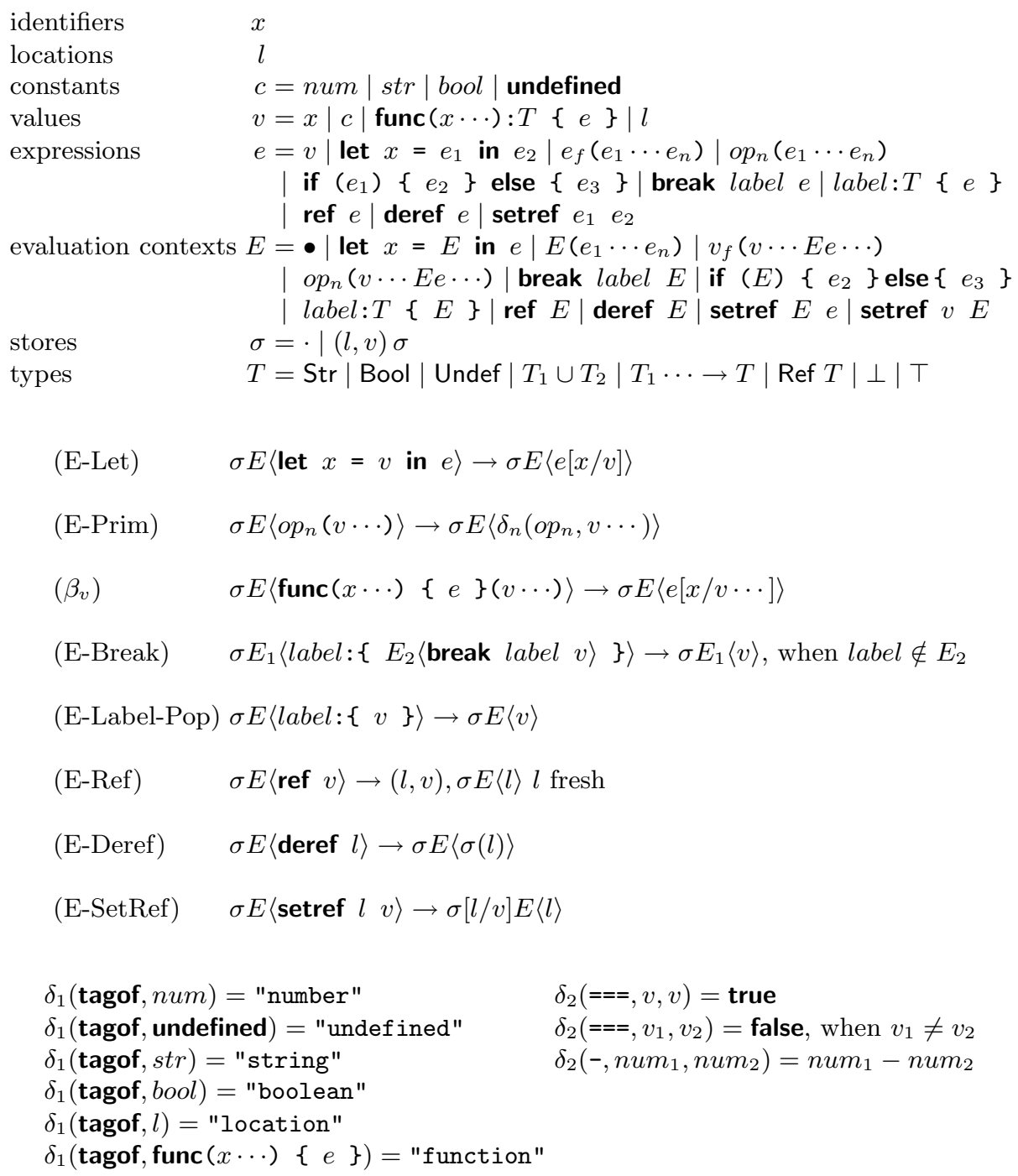

Fig. 4. Syntax and Semantics of $\lambda_{S}$

In this paper, the static types of $\lambda_{S}$ are much richer than its runtime tags. Therefore, we use a more technically precise name, tagof, to model the typeof operator of real scripting languages. The break operator can model both break and return statements of JavaScript. break aborts the current continuation up to a matching label, and returns a value. We specify the semantics of three primitives, of which physical equality (===) and tagof appear extensively in flow-directed 

(S-Refl) $T<: T$
(S-Trans) $\frac{S<: U \quad U<: T}{S<: T}$
(S-Bot) $\perp<: T$
(S-Top) $T<: \top$
$(\mathrm{S}-\mathrm{Arr}) \frac{S^{\prime}<: S \cdots \quad T<: T^{\prime}}{S \cdots \rightarrow T<: S^{\prime} \cdots \rightarrow T^{\prime}}$
(S-Ref) $\frac{T<: S \quad S<: T}{\operatorname{Ref} S<: \operatorname{Ref} T}$
$(\mathrm{S}-$ UnionE) $) \frac{S_{1}<: T \quad S_{2}<: T}{S_{1} \cup S_{2}<: T}$
$(\mathrm{S}-\mathrm{UnionL}) S<: S \cup T$
(S-UnionR) $T<: S \cup T$

Fig. 5. Subtyping in $\lambda_{S}$

$$
\begin{aligned}
& t y_{1}(\text { tagof })=\top \rightarrow \text { Str } \quad t y_{2}(===)=\top \times \top \rightarrow \text { Bool } \quad t y_{2}(-)=\text { Num } \times \text { Num } \rightarrow \text { Num } \\
& (\mathrm{T}-\mathrm{Loc}) \frac{\Sigma(l)=T}{\Sigma ; \Gamma \vdash l: T} \quad(\mathrm{~T}-\mathrm{Sub}) \frac{\Sigma ; \Gamma \vdash e: S \quad S<: T}{\Sigma ; \Gamma \vdash e: T} \\
& (\mathrm{~T}-\mathrm{Abs}) \frac{\Sigma ; \Gamma^{\prime}, x: S, \cdots \vdash e: T \quad \Gamma^{\prime}=\Gamma \text { with labels removed }}{\Sigma ; \Gamma \vdash \text { func }(x \cdots): S \cdots \rightarrow T\{e\}: S \cdots \rightarrow T} \\
& \text { (T-SetRef) } \frac{\Sigma ; \Gamma \vdash e_{1}: \operatorname{Ref} S \quad \Sigma ; \Gamma \vdash e_{2}: T \quad T<: S}{\Sigma ; \Gamma \vdash \text { setref } e_{1} e_{2}: \operatorname{Ref} T} \\
& \text { (T-If) } \frac{\Sigma ; \Gamma \vdash e_{1}: \text { Bool } \quad \Sigma ; \Gamma \vdash e_{2}: T \quad \Sigma ; \Gamma \vdash e_{3}: T}{\Sigma ; \Gamma \vdash \text { if }\left(e_{1}\right)\left\{e_{2}\right\} \text { else }\left\{e_{3}\right\}: T} \\
& \text { (T-Label) } \frac{\Sigma ; \Gamma, \text { label }: T \vdash e: T}{\Sigma ; \Gamma \vdash \text { label }: T\{e\}: T} \quad \text { (T-Break) } \frac{\Gamma(\text { label })=T \quad \Sigma, \Gamma \vdash e: T}{\Sigma ; \Gamma \vdash \text { break label } e: \perp}
\end{aligned}
$$

Fig. 6. Typing $\lambda_{S}$ (Essential Rules)

reasoning (figure11). Other expressions, such as tagof $\times$ !== "string", are a simple extension of our theory.

Figure 4 also specifies the syntax of types, $T$. Types include untagged unions and a top type $T$, which were motivated in section 2 . We also include the type of locations, Ref $T$, and a bottom type $\perp$ for control operators that do not return a value. Given these types, subtyping (figure 51) is conventional.

Our typing relation is also mostly conventional. We present select typing judgments in figure6. Note that the typing environment binds identifiers and labels. By T-SetRef, we can write subtypes to locations 2 Finally, like JavaScript, $\lambda_{S}$ programs cannot break across function boundaries, so we statically disallow it by dropping labels when typing functions (T-Abs).

$\overline{2}$ This is a simple restriction of source and sink types [20, Chapter 15.5]. 
$r=\{$ "string", "boolean", "number", "undefined", "function", "location" $\}$
$R=\mathcal{P}(r)$

$$
\begin{array}{ll}
\operatorname{runtime}: T \rightarrow R & \\
\operatorname{runtime}(\text { Str }) & =\{\text { "string" }\} \\
\operatorname{runtime}(\text { Bool }) & =\{\text { "boolean" }\} \\
\operatorname{runtime}(\text { Num }) & =\{\text { "number" }\} \\
\operatorname{runtime}(\text { Undef }) & =\{\text { "undefined" }\} \\
\operatorname{runtime}(S \cup T) & =\text { runtime }(S) \cup \text { runtime }(T) \\
\operatorname{runtime}(S \cdots \rightarrow T) & =\{\text { "function" }\} \\
\operatorname{runtime}(\perp) & =\emptyset \\
\operatorname{runtime}(\top) & =r \\
\operatorname{runtime}(\operatorname{Ref} T) & =\{\text { "location" }
\end{array}
$$

static $: R \times T \rightarrow T$

$\operatorname{static}(R, \operatorname{Str}) \quad=$ Str, if "string" $\in R$

$\operatorname{static}(R$, Bool $) \quad=$ Bool, if "boolean" $\in R$

$\operatorname{static}(R, \mathrm{Num}) \quad=$ Num, if "number" $\in R$

$\operatorname{static}(R$, Undef $) \quad=$ Undef, if "undefined" $\in R$

$\operatorname{static}(R, S \cdots \rightarrow T)=S \cdots \rightarrow T$, if "function" $\in R$

$\operatorname{static}(R, S \cup T) \quad=\operatorname{static}(R, S) \cup \operatorname{static}(R, T)$

$\operatorname{static}(R, S \cup T) \quad=\operatorname{static}(R, S)$, if $\operatorname{static}(R, T)$ is undefined

$\operatorname{static}(R, S \cup T) \quad=\operatorname{static}(R, T)$, if $\operatorname{static}(R, S)$ is undefined

$\operatorname{static}(R, \top) \quad=\top$

$\operatorname{static}(R, \operatorname{Ref} T) \quad=\operatorname{Ref} \top$

Fig. 7. Relationship Between Types and Tags

\section{Relating Static Types and Runtime Tags}

Consider the following JavaScript program:

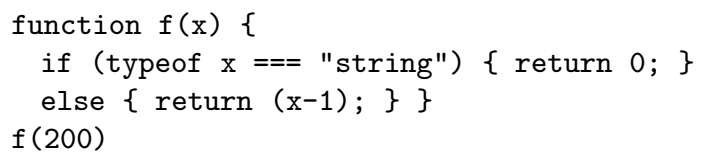

We can model this in $\lambda_{S}$ as follows, with $\mathrm{x}$ as a local variable and the breaks representing return statements and the intended type annotation inserted 3

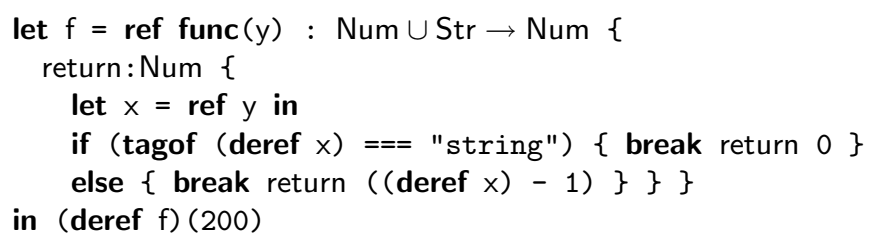

Both the $\lambda_{S}$ and original JavaScript programs run without error, returning 199.

${ }^{3}$ In earlier work, we desugared JavaScript to $\lambda_{J S}$ in this form 8 . 


$$
\begin{aligned}
e & =\cdots \mid \text { tagcheck } R e \mid \text { tagerr } \\
E & =\cdots \mid \text { tagcheck } R \quad E
\end{aligned}
$$

$\left(\right.$ E-TagCheck) $\frac{\delta_{1}(\text { tagof, } v) \in R}{\sigma E\langle\text { tagcheck } R v\rangle \rightarrow \sigma E\langle v\rangle}$

$\left(\right.$ E-TagCheck-Err) $\frac{\delta_{1}(\text { tagof, } v) \notin R}{\sigma E\langle\text { tagcheck } R v\rangle \rightarrow \sigma E\langle\text { tagerr }\rangle}$

$\left(\mathrm{T}\right.$-Check) $\frac{\Sigma ; \Gamma \vdash e: S \quad \operatorname{static}(R, S)=T}{\Sigma ; \Gamma \vdash \text { tagcheck } R e: T} \quad($ T-TagErr $) \Sigma ; \Gamma \vdash$ tagerr $: \perp$

Fig. 8. Typing and Evaluation of Checked Tags

This $\lambda_{S}$ program fails to type in the type checker of the previous section because - expects its operands to be numbers, but deref $x$ has type Num $\cup$ Str. However, the tag-test informs us, the reader, that $x$ has the static type Str in the true branch; the type annotation on y bounds its range of values, and thus enables us to conclude that $x$ has type Num in the false branch. Thus, the dynamic test and static type annotation collude to demonstrate that this program is statically safe. Our goal is to enable the static type checker to arrive at the same conclusion.

To support such reasoning, a retrofitted type system must relate static types and runtime tags. We show this in figure 7 runtime maps types to tag sets (due to the presence of unions), but since types are much richer than tags, we cannot distinguish all static types at runtime, e.g., all arrow types are mapped to the tag "function" (objects would be modeled similarly). static lets us narrow a type based on a known tag. For example, if a value has type $\operatorname{Str} \cup$ Num and its tag set is $\{$ "number" , then static produces the type Num. Note that static is partial: for example, static ( $\{$ "number" $\}$, Str) is undefined.

Since static relates types and tags, our type system can use it to account for runtime tag-tests. We use static by extending $\lambda_{S}$ with an auxiliary construct, tagcheck $R e$ (figure 8), which narrows the type of $e$ based on the tag set $R$. By judiciously inserting tagchecks, we can make our example typable 4 We thus offer tagcheck as an appropriate cast-like operator for scripting languages.

A tagcheck expression can fail in three ways. Two are static: when the tag set $R$ is incompatible with the type of $e$, static is undefined; even if it is compatible, the resulting type may not be what the context expects. However, the third failure is dynamic: if $e$ reduces to $v$ and $\operatorname{tagof}(v) \notin R$, then evaluation gets stuck with a tagerr (E-TagCheck-Err). This error condition manifests itself when we try to prove a type soundness theorem.

\footnotetext{
${ }^{4}$ Section 5 presents an efficient technique to insert tagchecks automatically, so they are hidden from the programmer.
} 
The preservation lemma is conventional:

Lemma 1 (Preservation) If $\Sigma, \vdash e: T, \Sigma \vdash \sigma$, and $\sigma e \rightarrow \sigma^{\prime} e^{\prime}$, then there exists a $\Sigma^{\prime}$, such that:

i. $\Sigma^{\prime}, \cdot \vdash \sigma^{\prime} e^{\prime}: T$, and

ii. $\Sigma \subseteq \Sigma^{\prime}$.

However, programs can get stuck on tagerrs:

Lemma 2 (Progress) If $\Sigma, \vdash e: T$ and $\Sigma \vdash \sigma$, then either:

i. $e \in v$, or

ii. there exist $\sigma^{\prime}$ and $e^{\prime}$, such that $\sigma e \rightarrow \sigma^{\prime} e^{\prime}$, or

iii. $e=E\langle$ tagerr $\rangle$, for some $E$.

Thus, the type soundness theorem is unsatisfying because of (iii.) of the lemma above. We could try to "repair" the type system; indeed, a sufficiently complicated type system might not need tagchecks and tagerrs at all. Our key idea is to admit tagerrs to keep the type system simple, and then discharge them by other means.

\section{Automatically Inserting Safe tagchecks}

We need a way to automatically insert tagchecks that fail neither statically nor at runtime. The tagcheck-insertion technique needs to be sound and handle uses of local control and state that we presented in section 2. Unlike conventional type systems, flow analyses are well-suited to such reasoning styles, so we consider flow analysis here. Unfortunately, whole-program analysis of functional and objectoriented languages is non-modular and expensive (section 7). Moreover, we need to relate abstract heaps produced by flow analysis to types produced by typechecking. We address these problems broadly, before formally presenting one particular analysis (section [6).

The goal of the flow analysis is to compute the tag-sets necessary for tagcheck expressions. Therefore, the domain of the analysis will be tag-sets augmented by some book-keeping information. Returning to the example from section 4, the comments illustrate the kind of information we need from flow analysis:

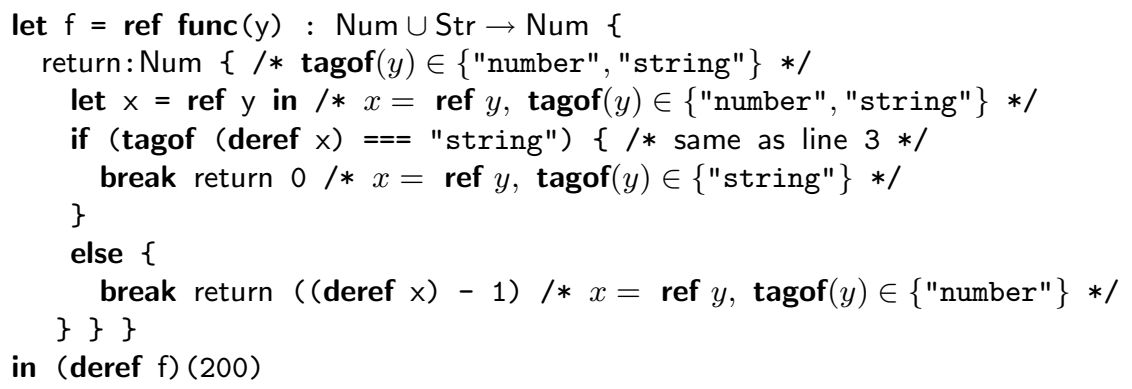


The flow analysis should compute that $x=$ ref $y$ at all program points, and that on lines 4 and $8, \operatorname{tagof}(y) \in\{$ "number", "string" $\}$ and tagof $(y) \in\{$ "number" $\}$, respectively. This information is enough to mechanically transform the program, replacing the (deref $x$ ) expressions with tagcheck $\{$ "number", "string" $\}$ (deref $x$ ) on line 4 and tagcheck \{"string" $\}$ (deref $x$ ) on line 8. Section 6 details a controlsensitive, heap-sensitive analysis that produces results such as this.

This analysis, like our type system, is mostly conventional. It is peculiar in populating the initial abstract heap with $\operatorname{tagof}(y) \in\{$ "number", "string" $\}$. A whole-program analysis might have used the application on line 10 to populate the heap with the argument value of 200. In contrast, our analysis remains local but exploits the type annotation on $y$, thus determining that $\operatorname{tagof}(y)$ is in runtime $($ Num $\cup$ Str $)=\{$ "number", "string" $\}$.

We thus use types to modularize our flow analysis, so the analysis can remain strictly intraprocedural. The time complexity of flow analysis is therefore a function of the size of individual functions in the program, which does not tend to grow as programs get larger. (Of course, the choice of function calls as modularity boundaries is not essential.) However, this does reduce precision, as we see below.

Assignment and Aliasing. Our analysis is locally heap-sensitive and can typecheck the following imperative variant of the example function:

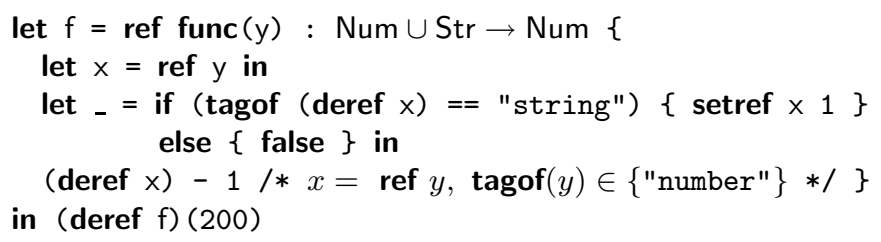

However, since we restart the analysis at function applications, we do not track non-local effects. In the following example, since foo $(x)$ may assign either a number or a string to $x$, the analysis we present in section 6 simply restarts on all function applications. Thus we cannot insert a useful tagcheck around the subsequent deref $x$, so the example is untypable:

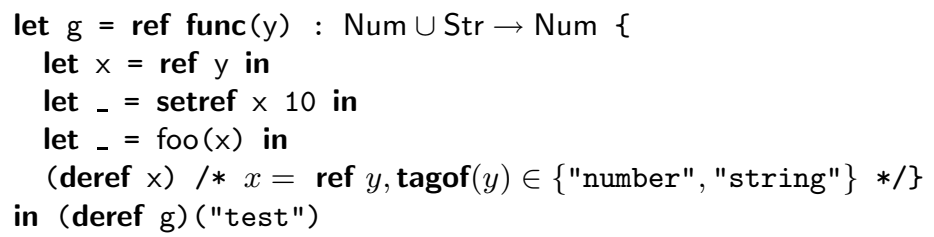

More sophisticated analyses that tracked ownership or aliasing could make such examples typeable.

Soundness. Given that our flow analysis ignores actual arguments, is it sound? To show that a flow analysis is sound, we must define an acceptability relation and prove that statically computed abstract heaps remain acceptable under evaluation. However, here is a trivial variation of our example that violates acceptability: 


\begin{tabular}{|l|c|c|c|}
\hline & JavaScript & Python & Ruby \\
\hline Loops & $\checkmark$ & $\checkmark$ & $\checkmark$ \\
Exceptions & $\checkmark$ & $\checkmark$ & $\checkmark$ \\
Generators & & $\checkmark$ & $\checkmark$ \\
Labelled Statements & $\checkmark$ & & \\
Switch fall-through & $\checkmark$ & & \\
Continuations & & & $\checkmark$ \\
\hline
\end{tabular}

Fig. 9. Control Features of Scripting Languages

values

$\begin{array}{cc}\text { binding expressions } \quad B & =V \mid \text { ref } V \mid \text { deref } V \mid \text { setre } \\ \mid & \text { tagcheck } R V \mid \text { tagerr }\end{array}$

$V=x|c| l|\operatorname{func}(x \cdots): T\{M\}| \operatorname{func}(x \cdots)\{M\}$

unlabeled expressions $N=$ let $x=B$ in $M \mid V_{f}(V \cdots)$

labelled expressions $\quad M=N^{\hat{\imath}}$

if $(V)\left\{M_{1}\right\}$ else $\left\{M_{2}\right\}$

stores $\quad S=\cdot \mid(l, V) S$

Fig. 10. Syntax of $\lambda_{S}$ in CPS

let $f=\operatorname{ref}$ func $(y): \operatorname{Num} \cup \operatorname{Str} \rightarrow \operatorname{Num}\{/ * \ldots$ as before $\ldots * /\}$

in (deref $f$ ) (true)

The flow analysis ignores the actual argument true (tagged "boolean") and instead assumes that the type annotation is correct. That is, it assumes that at runtime, y is tagged either "number" or "string". Thus, we obtain only a weak soundness lemma (lemma 4).

Although flow analysis admits such mis-applied functions, the type system ensures that function applications are well-typed. Conversely, although the type system admits tagerrs at runtime, the flow analysis only inserts tagchecks that provably do not produce tagerrs. Hence, each component resolves the other's weakness and in concert they combine to statically check programs that they cannot verify alone.

\section{Flow Analysis via CPS}

A glaring issue with $\lambda_{S}$ is that it has a single control operator, while real scripting languages support a plethora of control operators (figure 9). To avoid presenting an overly break-specific program analysis, we convert $\lambda_{S}$ to CPS. CPS has the added advantage of naming intermediate terms, thereby simplifying our analysis. CPS is, however, not a requirement; we only use it for convenience.

\subsection{CPS Transformation}

Figure 10 specifies the syntax of CPS- $\lambda_{S}$, which, with the exception of $V$, is a syntactic restriction of $\lambda_{S} . V$ includes administrative functions (explained shortly). 


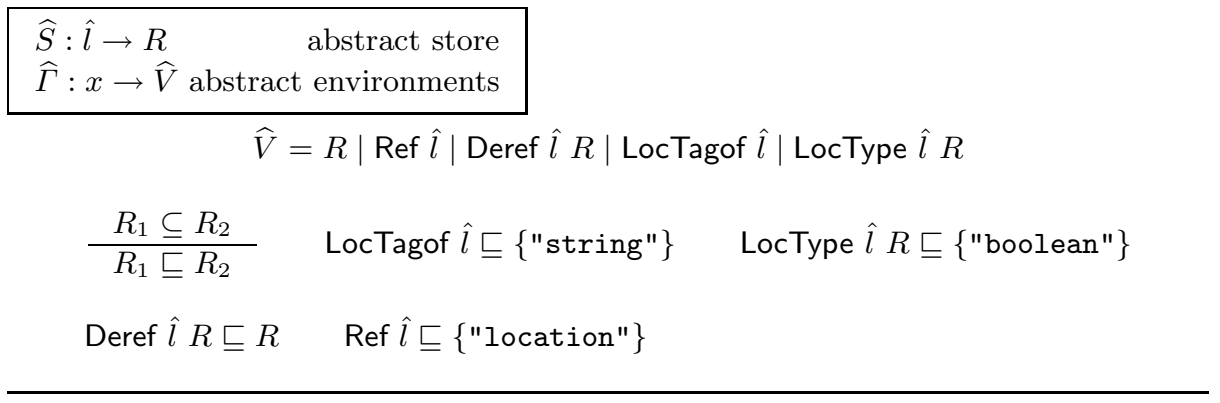

Fig. 11. Analysis Domains

We specify the CPS transformation using a technique developed by Sabry and Felleisen [21. The transformation is defined by four mutually-recursive functions that respectively map programs, expressions, values, and evaluation contexts from direct-style to CPS:

$$
\mathcal{P}_{k}: \sigma e \rightarrow S M \quad \Phi: v \rightarrow V \quad \mathcal{C}_{k}: e \rightarrow M \quad \mathcal{K}_{k}: E \rightarrow V
$$

For illustration, consider representative cases of these functions:

$$
\begin{aligned}
\mathcal{P}_{k} \llbracket(l, v) \cdots e \rrbracket & =(l, \Phi(v)) \cdots \mathcal{C}_{k} \llbracket e \rrbracket \\
\Phi \llbracket \operatorname{func}(x \cdots): S \cdots \rightarrow T\{e\} \rrbracket & =\text { func }(k, x \cdots):(T \rightarrow \perp) \times S \cdots \rightarrow \perp\left\{\mathcal{C}_{k} \llbracket e \rrbracket\right\} \\
\mathcal{C}_{k} \llbracket E\left\langle v_{f}\left(v_{\text {arg }} \cdots\right)\right\rangle \rrbracket & =\Phi \llbracket v_{f} \rrbracket\left(\mathcal{K}_{k} \llbracket E \rrbracket, \Phi \llbracket v_{\text {arg }} \rrbracket \cdots\right) \\
\mathcal{K}_{k} \llbracket E\langle\text { let } x=\bullet \text { in } e\rangle \rrbracket & =\text { func }(x)\left\{\mathcal{C}_{k} \llbracket E\langle e\rangle \rrbracket\right\}
\end{aligned}
$$

In the last case above, the transformation introduces functions not found in the source program to receive the bound value. Since all evaluation contexts are transformed into such "administrative" functions, all control structures are thus transformed into applications of administrative functions.

For succinctness, we do not introduce continuation-passing operators, and instead let-bind operators' results. We elide the semantics of CPS- $\lambda_{S}$, since it is essentially the same as the semantics in figure 4. This style of definition makes it easy to prove that direct-evaluation corresponds to CPS-evaluation, which is necessary to relate typing and flow analysis.

Lemma 3 (Soundness of CPS Transformation) If $\sigma e \rightarrow \sigma^{\prime} e^{\prime}$ using reduction rule $R$, then $\mathcal{P}_{k} \llbracket \sigma e \rrbracket \rightarrow \mathcal{P}_{k} \llbracket \sigma^{\prime} e^{\prime} \rrbracket$ using reduction rules $R$, E-Let, and $\widehat{\beta_{v}}$.

In the lemma above, $\widehat{\beta_{v}}$ denotes the reduction rule for administrative functions (defined exactly as $\beta_{v}$ ). The lemma roughly states that intermediate redexes in CPS are applications of administrative functions and let-expressions.

\subsection{Modular Flow Analysis}

Figure 11 specifies our abstract values and the lattice that relates them. Abstract stores $(\widehat{S})$ map abstract locations $(\hat{l})$ to tag sets $(R)$. (Abstract locations 


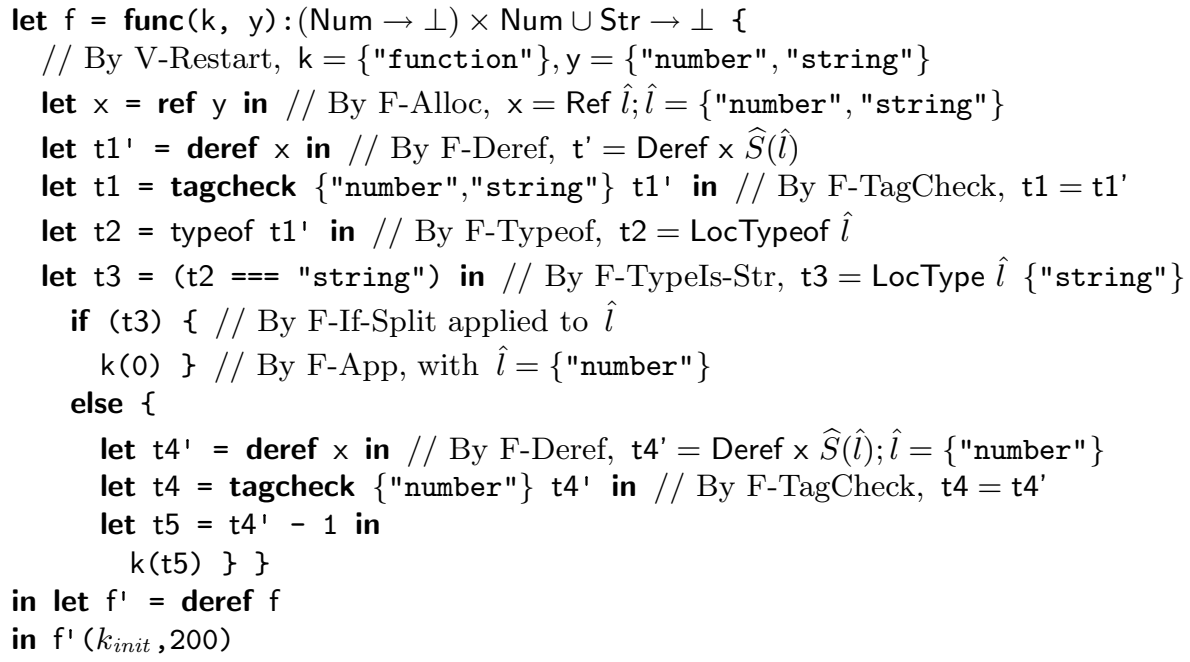

Fig. 12. tagcheck Insertion

are labels on expressions, introduced by CPS.) On the other hand, abstract environments $(\widehat{\Gamma})$ map identifiers to abstract values $(\widehat{V})$ that will account for tag-tests.

For example, figure 12 presents our example from the previous section in CPS. The comment on line 2 specifies the initial abstract environment, computed by applying runtime to the arguments. The remaining comments specify how the abstract heap and environment are transformed by each statement. These transformation are acceptable, as specified by our acceptability relation (figure 13).

Note that the user-written identifier $\mathrm{x}$ is bound to a heap-location. However, the CPS-introduced identifiers, which name the subexpressions that reason about $\mathrm{x}$, are not heap-allocated. We exploit this stratification in our analysis domains to simplify the proof of soundness. The abstract heap and environment contain values that locally reason about the heap. For soundness, V-Restart therefore discards the abstract heap and uses reset and del to widen heap-dependent abstract values to simple tag sets.

Assignment and Aliasing. In figure 14, we account for the effects of assignments to tag sets. If a program sets an abstract location $\hat{l}$, then F-SetRef simply updates $\hat{l}$ in the abstract store of its continuation. However, the environment may bind identifiers to abstract values that reason about $\hat{l}$. Therefore, we use del to widen $\hat{l}$-dependent values to simple tag sets.

Local variables cannot reference each other. However, we use references to model mutable objects as well. A local variable bound to a mutable object is a reference to a reference, and these objects can be aliased. In these cases, we stop tracking the potentially-aliased abstract location, once again using del. 


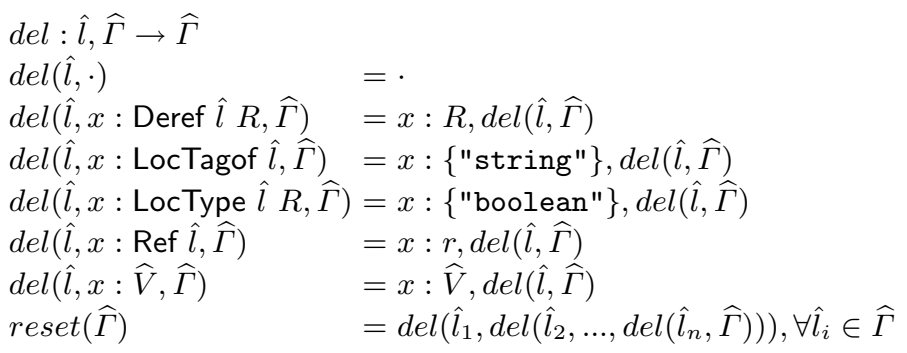

$\widehat{\Gamma} \triangleright V \rightsquigarrow \widehat{V}$

$($ V-Restart $) \frac{; x: \operatorname{runtime}(T) \cdots, \operatorname{reset}(\widehat{\Gamma}) \vDash M}{\widehat{\Gamma} \triangleright \text { func }(x \cdots): T \cdots \rightarrow \perp\{M\} \rightsquigarrow \text { "function" }}$

(V-Const) $\widehat{\Gamma} \triangleright c \rightsquigarrow \delta_{1}(, c) \quad(\mathrm{V}-\mathrm{Id}) \widehat{\Gamma} \triangleright x \rightsquigarrow \widehat{\Gamma}(x)$

$(\mathrm{V}-\mathrm{Sub}) \frac{\widehat{\Gamma} \triangleright V \rightsquigarrow \widehat{V} \widehat{V} \sqsubseteq \widehat{V^{\prime}}}{\widehat{\Gamma} \triangleright V \rightsquigarrow \widehat{V^{\prime}}}$

$\widehat{S} ; \widehat{\Gamma} \vDash M$

(F-LetVal) $\frac{\widehat{S} \triangleright V \rightsquigarrow \widehat{V} ; x: \widehat{V}, \widehat{\Gamma} \vDash M}{\widehat{S} ; \widehat{\Gamma} \vDash \text { let } x=V \text { in } M} \quad$ (F-Alloc) $\frac{\hat{l}: R, \widehat{S} ; x: \operatorname{Ref} \hat{l}, \widehat{\Gamma} \vDash M}{\widehat{S} ; \widehat{\Gamma} \vDash \operatorname{let}^{\hat{\imath}} x=\operatorname{ref} V \text { in } M}$

(F-Deref) $\frac{\widehat{\Gamma} \triangleright V \rightsquigarrow \operatorname{Ref} \hat{l} \quad \widehat{S}(\hat{l})=R \quad \widehat{S} ; x: \text { Deref } \hat{l} R, \widehat{\Gamma} \vDash M}{\widehat{S} ; \widehat{\Gamma} \vDash \text { let } x=\text { deref } V \text { in } M}$

(F-Tagof) $\frac{\widehat{\Gamma} \triangleright V \rightsquigarrow \text { Deref } \hat{l} R \quad \widehat{S} ; x: \text { LocTagof } \hat{l}, \widehat{\Gamma} \vDash M}{\widehat{S} ; \widehat{\Gamma} \vDash \text { let } x=\text { tagof } V \text { in } M}$

(F-TypeIs-Str) $\sqrt{a} \frac{\widehat{\Gamma} \triangleright V \rightsquigarrow \text { LocTagof } \hat{l} \quad \widehat{S} ; x \text { : LocType } \hat{l}\{\text { "string" }\}, \widehat{\Gamma} \vDash M}{\widehat{S} ; \widehat{\Gamma} \vDash \text { let } x=V \text { === "string"in } M}$

(F-TagCheck) $\frac{\widehat{\Gamma} \triangleright V \rightsquigarrow R \quad \widehat{S} ; x: R, \widehat{\Gamma} \vDash M}{\widehat{S} ; \widehat{\Gamma} \vDash \text { let } x=\text { tagcheck } R V \text { in } M}$

(F-If-Split) $\frac{\widehat{\Gamma} \triangleright V \rightsquigarrow \text { LocType } \hat{l} R \quad \widehat{S}[\hat{l}:=R] ; \widehat{\Gamma} \vDash M_{1} \quad \widehat{S}[\hat{l}:=\widehat{S}(\hat{l}) \backslash R] ; \widehat{\Gamma} \vDash M_{2}}{\widehat{S} ; \widehat{\Gamma} \vDash \text { if }(V)\left\{M_{1}\right\} \text { else }\left\{M_{2}\right\}}$ (F-App) $\frac{\widehat{\Gamma} \triangleright V_{f} \rightsquigarrow \widehat{V_{f}} \quad \widehat{\Gamma} \triangleright V \rightsquigarrow R \cdots}{\widehat{S} ; \widehat{\Gamma} \vDash V_{f}(V \cdots)}$

$\overline{{ }^{a} \text { F-TypeIsStr }}$ is easily generalized to arbitrary tags; we specialize it to strings for presentation only.

Fig. 13. Acceptability of Flow Analysis (Essential Rules) 


$$
\begin{aligned}
& \text { (F-SetRef) } \frac{\widehat{\Gamma} \triangleright V_{1} \rightsquigarrow \operatorname{Ref} \hat{l} \quad \widehat{\Gamma} \triangleright V_{2} \rightsquigarrow R \quad \widehat{S}[\hat{l}:=R] ; x: \operatorname{Ref} \hat{l}, \operatorname{del}(\hat{l}, \widehat{\Gamma}) \vDash M}{\widehat{S} ; \widehat{\Gamma} \vDash \text { let } x=\text { setref } V_{1} V_{2} \text { in } M} \\
& (\text { F-Ref-Alias }) \frac{\widehat{\Gamma} \triangleright V \rightsquigarrow \operatorname{Ref} \hat{l} \quad \widehat{S} ; x: r, \operatorname{del}(\hat{l}, \widehat{\Gamma}) \vDash M}{\widehat{S} ; \widehat{\Gamma} \vDash \text { let } x=\operatorname{ref} V \text { in } M}
\end{aligned}
$$

Fig. 14. Assignment and Aliasing

F-Ref-Alias in figure 14 tackles aliasing in ref expressions. Similar rules apply to other syntactic forms.

Monotone Framework. Our algorithm for computing tagchecks is a simple monotone framework 15. directly derived from the rules in figure 13. The monotone framework computes the abstract store and environment at each labelled expression. We use this information to insert tagchecks into our programs.

Consider each expression of the form:

$$
\text { let }^{\hat{l}} r=\text { deref } x \text { in } M
$$

Let $\widehat{\Gamma}$ and $\widehat{S}$ be the computed abstract environment and store at $\hat{l}$. If $\widehat{\Gamma}(\hat{l})=$ Ref $\hat{l}^{\prime}$, then we transform the expression to:

$$
\begin{aligned}
& \text { let } r^{\prime \hat{l}}=\text { deref } x \text { in } \\
& \text { let } r=\operatorname{tagcheck} \widehat{S}\left(\hat{l}^{\prime}\right) r^{\prime} \text { in } \\
& M
\end{aligned}
$$

For type-checking, this inserted tagcheck is mapped back to the original, directstyle program.

The administrative functions, if applied, can exponentially increase the size of programs. Therefore, we leave certain administrative redexes unapplied (e.g., continuations of if-expressions). The CPS transformation is therefore linear time and our flow analysis computes meets through administrative functions.

Complexity. Our flow analysis is a monotonic ascent of a lattice of finite height. For a program of $N$ terms our analysis computes an abstract store and environment at each term. The domain of abstract stores and environments are both of size $O(N)$. The range of the abstract store is $R$, and $|R|$ is a constant. The range of the abstract environment is $\widehat{V}$, where $\widehat{V}$ contains the elements of $R$. The additional elements of $\widehat{V}$ are incomparable with each other and are all less than the elements of $R$. Hence, the height of $\widehat{V}$ is just 1 greater than the height of $R$. Thus, the analysis needs time quadratic in the program size. In practice, our prototype implementation type-checks real-world JavaScript programs in seconds on modest machines.

Soundness. In addition to figure 13 and figure 14, we require trivial rules for cases where our flow analysis cannot determine useful information. These additional 
rules admit all other expressions, except tagerrs and possibly-faulty tagchecks. Soundness also requires auxiliary rules that reason about the concrete values in the store that are introduced by evaluation. We elided the concrete store from figures 13 and 14 for clarity; in the following lemmas, we introduce it.

Lemma 4 (Soundness) If $\hat{S}, \cdot \vDash S M$ and $S M \rightarrow S^{\prime} M^{\prime}$ then either:

i. $\hat{S}^{\prime}, \cdot \vDash S^{\prime} M^{\prime}$, or

ii. $M$ is a $\beta_{v}$-redex, func $(x \cdots): T \cdots \rightarrow \perp\{N\}(V \cdots)$, where for some $V$, $\delta_{1}($ tagof, $V) \notin$ runtime $(T)$.

\subsection{Combining Typing and Flow Analysis}

We can now prove a stronger progress result that eliminates tagerrs.

\section{Theorem 1 (Strengthened Progress) If:}

i. $\Sigma ; \cdot \vdash e: T$,

ii. $\Sigma \vdash \sigma$, and

iii. $\widehat{S} ; \cdot \vDash \mathcal{P}_{k} \llbracket \sigma e \rrbracket$,

then either:

i. $e \in v$, or

ii. There exist $\sigma^{\prime}$ and $e^{\prime}$, such that $\sigma e \rightarrow \sigma^{\prime} e^{\prime}$.

Proof: This follows from lemma 2, with the possibility of tagerrs eliminated by inspection of figure 13 flow analysis does not admit expressions with tagerrs.

Theorem 1 requires a corresponding, combined preservation theorem.

\section{Theorem 2 (Combined Preservation) If:}

i. $\Sigma ; \cdot \vdash e: T$,

ii. $\Sigma \vdash \sigma$,

iii. $\widehat{S} ; \cdot \vDash \mathcal{P}_{k} \llbracket \sigma e \rrbracket$, and

iv. $\sigma e \rightarrow \sigma^{\prime} e^{\prime}$,

then there exist $\Sigma^{\prime}$ and $\widehat{S}^{\prime}$, such that:

i. $\Sigma^{\prime} ; \cdot \vdash e^{\prime}: T$,

ii. $\Sigma^{\prime} \vdash \sigma^{\prime}$,

iii. $\Sigma \subseteq \Sigma^{\prime}$, and

iv. $\hat{S}^{\prime} ; \cdot \vDash \mathcal{P}_{k} \llbracket \sigma^{\prime} e^{\prime} \rrbracket$.

Proof: Conclusions (i.), (ii.), and (iii.) follow immediately from lemma 1 For conclusion (iv.), apply lemma 3 to hypothesis (iv.) to get a reduction sequence, $\mathcal{P}_{k} \llbracket \sigma e \rrbracket \rightarrow \mathcal{P}_{k} \llbracket \sigma^{\prime} e^{\prime} \rrbracket$. Apply lemma 4 at each step, eliminating case (ii.) of the lemma as follows. By lemma 3 intermediate expressions are not $\beta_{v}$-redexes, so case (ii.) does not apply. Suppose $e$ itself has an active $\beta_{v}$-redex:

$$
e=E\left\langle\operatorname{func}(x \cdots): U \cdots \rightarrow S\left\{e_{f}\right\}(v \cdots)\right\rangle
$$




$$
\begin{array}{ccc}
\Gamma \vdash e_{1}: \tau_{1} ; \phi_{1} & \Gamma+\phi_{1} \vdash e_{2}: \tau_{2} ; \phi_{2} \quad \Gamma-\phi_{1} \vdash e_{3}: \tau_{3} ; \phi_{3} \\
\vdash \tau_{2}<: \tau & \vdash \tau_{3}<: \tau \quad \phi=\operatorname{combpred}\left(\phi_{1}, \phi_{2}, \phi_{3}\right)
\end{array}
$$

Fig. 15. If-splitting in Typed Scheme [23]

Once transformed to CPS, $e$ has the form

$$
\operatorname{func}(k, x \cdots):(S \rightarrow \perp) \times U \cdots \rightarrow \perp\left\{M_{p}\right\}(V \cdots)
$$

where $V \cdots$ are $v \cdots$ in CPS. Since $e$ is typed, there exists a $\Gamma$ such that:

$$
\Sigma ; \Gamma \vdash \operatorname{func}(x \cdots): U \cdots \rightarrow S\left\{e_{f}\right\}(v \cdots): S
$$

For all $v, \Sigma ; \Gamma \vdash v: U$ by inversion. Hence $\delta_{1}$ (tagof, $\left.v\right) \in \operatorname{runtime}(U)$. Since conversion to CPS does not change tags, $\delta_{1}$ (tagof, $\left.v\right)=\delta_{1}$ (tagof, $V$ ), case (ii.) of lemma 4 does not apply.

\section{Related Work}

Typed Scheme. Typed Scheme [2324] is a type system designed to admit Scheme idioms. Typed Scheme uses occurrence typing to account for type tests and type predicates. However, occurrence typing is unsound in the presence of imperative features; thus, it is "turned off" when imperative features are used. Unlike the Scheme programs that Typed Scheme types, programs in mainstream scripting languages make heavy use of imperative features, which we handle.

Technically, we develop a type system and flow analysis that are complementary by design (Lemmas 2 and 4), which combine soundly (Theorems 1 and 2), and which can be enriched independently within the framework of these two lemmas. We conjecture that a similar structure could be extracted from Typed Scheme, as the type system is augmented with meta-functions that update the environment (see Typed Scheme's use of $\Gamma+$ and $\Gamma$ - to affect the environment, and combpred to prop type tests to the context in if (figure 15)). We believe these are similar to transfer functions for dataflow analyses. However, Typed Scheme is not organized in this manner.

Intensional Polymorphism. Intensional polymorphism 2] provides a typecase construct that allows programs to inspect and dispatch on the type of values at runtime. This requires a term-level representation of types at runtime, which is only possible when the static and dynamic semantics of a programming language are co-designed. The present work, Typed Scheme, and other retrofitted type systems (discussed below) do not have access to their types at runtime. Type dispatch in a retrofitted type system happens indirectly. For example, Typed Scheme uses predicates [23, while our work relies on the relationship between static types and runtime tags (section 4). 
Other Retrofitted Type Systems. Soft Scheme 25] performs type inference for Scheme programs. It handles the full language of the time, and has a limited form of if-splitting. It does not pay any additional attention to the interaction of types and control flow. This is reasonable because it, like Typed Scheme, is focused on Scheme programs that are mostly functional. However, this means that it too cannot handle the kinds of examples shown in this paper and found in many scripting languages.

Anderson et al. [1] tackle type inference for JavaScript. However, their language is extremely limited, and their type system cannot tackle the idioms discussed in this paper (section 2).

Heidegger and Thiemann's [10] recency types account for ad hoc object initialization patterns that are pervasive in JavaScript, but does not address the problems that this paper does. Our work does not account for objects. Our preliminary investigation suggests that the two approaches are complementary and can fruitfully be combined.

Henglein and Rehof 12 present a translation of Scheme to ML that uses type inference to minimize runtime projections. However, their "type system does not model control flow information" [12, Section 6.5], which is the goal of our work.

Diamondback Ruby [5] is a type system and type inference for Ruby. Although its type language includes union types, it does not account for type-tests to discriminate members of unions, which is the focus of our work. The authors state that "support for occurrence types would be useful future work".

Types and Flow Analysis. Shivers shows how control-flow can be extended to account for type-tests [22, Chapter 9]. However, whole-program analysis for functional and object-oriented languages is non-modular and expensive 4] or difficult to make effective [3. Meunier et al. [16] develops a modular analysis for an untyped language by using contracts as sources and sinks for abstract values. We exploit type annotations in the same manner. However, unlike contracts, which are assumed to be correct, typing ensures that type annotations are correct. Since all functions have type annotations, our flow analysis problem is significantly more tractable than in an untyped language with optional contracts.

Jensen et al. 1314] and MrSpidey [4] use flow analysis to recover precise typelike information for arbitrary JavaScript and Scheme programs, respectively. A significant advantage of flow analysis is that it does not require type annotations. Our work requires and exploits type annotations to achieve modularity, which leads to quadratic time complexity in theory that appears to translate into practice (section 6.2).

There are known equivalences between various type systems and controlflow analyses, e.g., Heintze [11, Nielson and Nielson [18], and Palsberg and O'Keefe [19]. The aforementioned works extend type systems to calculate information that is conventionally calculated by flow analyses. In contrast, our type system is oblivious to control flow information (figure 6). We use a separate flow analysis to account for control-sensitive and heap-sensitive reasoning (section 5). We independently prove typing and flow analysis sound, then show that they combine in a simple way (section 6.3). 
Definite assignment analysis is a commonly used flow analysis that augments typing (e.g., see the Java Language Specification [7, Chapter 16]). Definite assignment analysis conservatively ensures that variables are assigned before they are used. Hence, the analysis rejects programs as untypable when all variables are not definitely assigned. In contrast, our analysis augments the type system to accept programs that would otherwise be untypable.

\section{Acknowledgements}

We thank Nicholas Cameron, Matthias Felleisen, and the anonymous reviewers for their careful comments on earlier drafts. We also thank Gilad Bracha, Cormac Flanagan, Jasvir Nagra, Steven Reiss, Ankur Taly, and Jan Vitek for enlightening discussions. This work is partially supported by the NSF and by Google.

\section{References}

1. Anderson, C., Giannini, P., Drossopoulou, S.: Towards type inference for javaScript. In: Gao, X.-X. (ed.) ECOOP 2005. LNCS, vol. 3586, pp. 428-452. Springer, Heidelberg (2005)

2. Crary, K., Weirich, S., Morrisett, G.: Intentional polymorphism in type-erasure semantics. In: ACM SIGPLAN International Conference on Functional Programming (1998)

3. Flanagan, C., Felleisen, M.: Componential set-based analysis. In: ACM SIGPLAN Conference on Programming Language Design and Implementation (1997)

4. Flanagan, C., Flatt, M., Krishnamurthi, S., Weirich, S., Felleisen, M.: Catching bugs in the web of program invariants. In: ACM SIGPLAN Conference on Programming Language Design and Implementation (1996)

5. Furr, M., An, J.D., Foster, J.S., Hicks, M.: Static type inference for Ruby. In: ACM Symposium on Applied Computing (2009)

6. Google JavaScript style guide, http://google-styleguide.googlecode.com/svn/trunk/javascriptguide.xml

7. Gosling, J., Joy, B., Steele, J.G.L., Bracha, G.: The Java Language Specification, 3rd edn. Addison Wesley, Reading (2005)

8. Guha, A., Saftoiu, C., Krishnamurthi, S.: The essence of javaScript. In: D'Hondt, T. (ed.) ECOOP 2010. LNCS, vol. 6183, pp. 126-150. Springer, Heidelberg (2010)

9. Harper, R., Mitchell, J.C.: On the type structure of Standard ML. ACM Transactions on Programming Languages and Systems 15(2) (1993)

10. Heidegger, P., Thiemann, P.: Recency types for dynamically-typed, object-based languages: Strong updates for JavaScript. In: ACM SIGPLAN International Workshop on Foundations of Object-Oriented Languages (2009)

11. Heintze, N.: Control-flow analysis and type systems. In: International Static Analysis Symposium (1995)

12. Henglein, F., Rehof, J.: Safe polymorphic type inference for a dynamically typed language: Translating Scheme to ML. In: ACM SIGPLAN International Conference on Functional Programming (1995)

13. Jensen, S.H., Møller, A., Thiemann, P.: Type analysis for JavaScript. In: International Static Analysis Symposium (2009) 
14. Jensen, S.H., Møller, A., Thiemann, P.: Interprocedural analysis with lazy propagation. In: International Static Analysis Symposium (2010)

15. Kildall, G.A.: A unified approach to global program optimization. In: ACM SIGPLAN-SIGACT Symposium on Principles of Programming Languages (1973)

16. Meunier, P., Findler, R.B., Felleisen, M.: Modular set-based analysis from contracts. In: ACM SIGPLAN-SIGACT Symposium on Principles of Programming Languages (2006)

17. Mikhajlov, L., Sekerinski, E.: A study of the fragile base class problem. In: European Conference on Object-Oriented Programming (1998)

18. Nielson, F., Nielson, H.R.: Type and effect systems. In: Olderog, E.-R., Steffen, B. (eds.) Correct System Design. LNCS, vol. 1710, p. 114. Springer, Heidelberg (1999)

19. Palsberg, J., O'Keefe, P.: A type system equivalent to flow analysis. In: ACM SIGPLAN-SIGACT Symposium on Principles of Programming Languages (1995)

20. Pierce, B.C.: Types and Programming Languages. MIT Press, Cambridge (2002)

21. Sabry, A., Felleisen, M.: Reasoning about programs in continuation-passing style. LISP and Symbolic Computation 6(3) (1993)

22. Shivers, O.: Control-Flow Analysis of Higher-Order Languages. PhD thesis, Carnegie Mellon University (1991)

23. Tobin-Hochstadt, S., Felleisen, M.: The design and implementation of Typed Scheme. In: ACM SIGPLAN-SIGACT Symposium on Principles of Programming Languages (2008)

24. Tobin-Hochstadt, S., Felleisen, M.: Logical types for untyped languages. In: ACM SIGPLAN International Conference on Functional Programming (2010)

25. Wright, A.K., Cartwright, R.: A practical soft type system for Scheme. ACM Transactions on Programming Languages and Systems 19(1) (1997) 